\title{
Time-Dependent Diffeomorphisms as Quantum Canonical Transformations and the Time-Dependent Harmonic Oscillator
}

\author{
Ali Mostafazadeh* \\ Department of Mathematics, Koç University, \\ Istinye 80860, Istanbul, TURKEY
}

\begin{abstract}
Quantum canonical transformations corresponding to time-dependent diffeomorphisms of the configuration space are studied. A special class of these transformations which correspond to time-dependent dilatations is used to identify a previously unknown class of exactly solvable time-dependent harmonic oscillators. The CaldirolaKanai oscillator belongs to this class. For a general time-dependent harmonic oscillator, it is shown that choosing the dilatation parameter to satisfy the classical equation of motion, one obtains the solution of the Schrödinger equation. A simple generalization of this result leads to the reduction of the Schrödinger equation to a second order ordinary differential equation whose special case is the auxiliary equation of the LewisRiesenfeld invariant theory. Time-evolution operator is expressed in terms of a positive real solution of this equation in a closed form, and the time-dependent position and momentum operators are calculated.
\end{abstract}

PACS numbers: 03.65.Bz, 03.65.Ge

\section{Introduction}

It is well-known that in quantum mechanics the unitary transformations of the Hilbert space correspond to the canonical transformations of the classical mechanics. Unfortunately, these

*E-mail address: amostafazadeh@ku.edu.tr 
quantum canonical transformations are not as widely used as their classical counterparts. The purpose of this article is to study the class of quantum canonical transformations defined by

$$
\mathcal{U}:=\exp \left[\frac{i \epsilon(t)}{2}\{f(x), p\}\right]=\exp [i \epsilon(t) \sqrt{f(x)} p \sqrt{f(x)}]
$$

and demonstrate their utility in solving the Schrödinger equation for time-dependent harmonic oscillators.円

Time-dependent harmonic oscillators have been the subject of active research since 1940's [1]- 14. This is because of the long list of applications of this system in modelling a variety of physical phenomena. Some recent applications of the time-dependent harmonic oscillators are in the study of the motion of ions in a Paul trap [9], quantum mechanical description of highly cooled ions [10, and emergence of nonclassical optical states of light due to a time-dependent dielectric constant [1]].

An interesting property of time-dependent harmonic oscillators is that the solution of the Schrödinger equation for this system can be reduced to that of the corresponding classical equation of motion [3]. It turns out that this reduction may be performed using a canonical transformation of the form (1). More generally, it is shown that the Schrödinger equation may be reduced to the solution of a certain second order ordinary differential equation which involves a parameter $k^{2}$ with values $-1,0$, and 1 . Any positive real solution of this equation with any of the choices for $k^{2}$ yields the solution of the Schrödinger equation. For $k^{2}=0$, one obtains the classical equation of motion. For $k^{2}=1$, one finds the auxiliary equation of the invariant theory of Lewis and Riesenfeld [5].

The organization of the paper is as follows. In Section 2 general results regarding timedependent quantum canonical transformations (11) and their special case corresponding to $f(x)=x$ are presented. These are then used in Section 3 to treat time-dependent harmonic oscillators. Section 4 is devoted to the quantum canonical transformation which leads to the reduction of the Schrödinger equation to the classical equation of motion. The generalization of this result, its connection with the invariant theory, and the calculation of the evolution operator and the Heisenberg observables are also discussed in this section. Section 5 deals with the canonical transformations corresponding to time-dependent diffeomorphisms which

\footnotetext{
${ }^{1}$ In Eq. (1), $x$ and $p$ denote position and momentum operators, respectively, $\{$, $\}$ stands for the anticommutator of two operators, and $f$ and $\epsilon$ are arbitrary real-valued smooth functions.
} 
change the metric of the space. The conclusions are presented in Section 6.

\section{Time-dependent Quantum Diffeomorphisms and Di- latations}

Let us first recall the effect of a general time-dependent quantum canonical transformation $\mathcal{U}=\mathcal{U}(t)$ on the Hamiltonian $H=H(t)$ and the time-evolution operator $U=U(t)$, i.e., the relations

$$
\begin{aligned}
H(t) \rightarrow H^{\prime}(t) & =\mathcal{U}(t) H(t) \mathcal{U}^{\dagger}(t)-i \mathcal{U}(t) \dot{\mathcal{U}}^{\dagger}(t) \\
U(t) \rightarrow U^{\prime}(t) & =\mathcal{U}(t) U(t) \mathcal{U}^{\dagger}(0)
\end{aligned}
$$

where a dot means a time-derivative and $\hbar$ is set to unity. These equations are direct consequences of the requirement that the Schrödinger equation

$$
H(t) U(t)=i \dot{U}(t), \quad U(0)=1
$$

must be preserved under the action of $\mathcal{U}$. Note that under a time-dependent quantum canonical transformation the Hamiltonian undergoes an affine (non-linear) transformation. Hence, unlike the dynamics (the Schrödinger equation) the energy spectrum is not preserved.

Next let us study the effect of the transformation induced by (1). In order to compute the transformed Hamiltonian $H^{\prime}$, one must first explore the effect of $\mathcal{U}$ on the position and momentum operators. A rather lengthy calculation shows that

$$
\begin{aligned}
x \rightarrow x^{\prime} & :=\mathcal{U} x \mathcal{U}^{\dagger}=\mathcal{F}_{1}(x) \\
p \rightarrow p^{\prime} & :=\mathcal{U} p \mathcal{U}^{\dagger}=\frac{1}{2}\left\{\mathcal{F}_{2}(x), p\right\}=\sqrt{\mathcal{F}_{2}(x)} p \sqrt{\mathcal{F}_{2}(x)}
\end{aligned}
$$

where

$$
\mathcal{F}_{1}(x):=e^{\epsilon(t) f(x) \frac{d}{d x}} x, \quad \mathcal{F}_{2}(x):=f(x) e^{\epsilon(t) f(x) \frac{d}{d x}} f^{-1}(x)
$$

In the derivation of these formulae use is made of Baker-Campbell-Hausdorff formula:

$$
e^{A} B e^{-A}=B+[A, B]+\frac{1}{2 !}[A,[A, B]]+\cdots,
$$


and the identities

$$
\begin{aligned}
{\left[\frac{i}{2}\left\{f_{1}(x), p\right\}, f_{2}(x)\right] } & =f_{1}(x) \frac{d}{d x} f_{2}(x), \\
{\left[\frac{i}{2}\left\{f_{1}(x), p\right\}, \frac{i}{2}\left\{f_{2}(x), p\right\}\right] } & =\frac{i}{2}\left\{f_{3}(x), p\right\}, \quad f_{3}(x):=f_{1} \frac{d}{d x} f_{2}(x)-f_{2} \frac{d}{d x} f_{1}(x),
\end{aligned}
$$

where $f_{1}$ and $f_{2}$ are arbitrary differentiable functions.

Eqs. (7) and (8) show that $\frac{1}{2}\{f(x), p\}$ generate diffeomorphisms of $\mathbb{R}$. Consequently, the transformations (11) are quantum canonical transformations associated with time-dependent diffeomorphisms of the configuration space.

In view of Eqs. (2), (5) and (6), one has

$$
H^{\prime}=H^{\prime}\left(x^{\prime}, p^{\prime} ; t\right)=H\left(x^{\prime}, p^{\prime} ; t\right)-\frac{\dot{\epsilon}(t)}{2}\{f(x), p\} .
$$

Now let us concentrate on a subclass of quantum canonical transformations of the form (1) corresponding to the choice $f(x)=x$. In this case, Eqs. (5), (6), and (9) reduce to

$$
\begin{aligned}
x \rightarrow x^{\prime} & =e^{\epsilon(t)} x, \quad p \rightarrow p^{\prime}=e^{-\epsilon(t)} p, \\
H \rightarrow H^{\prime} & =H\left(x^{\prime}, p^{\prime} ; t\right)-\frac{\dot{\epsilon}(t)}{2}\{x, p\} .
\end{aligned}
$$

Hence this choice yields time-dependent dilatations of the configuration space.

For a Hamiltonian of the standard form

$$
H=\frac{p^{2}}{2 m(t)}+V(x, t),
$$

Eqs. (10) and (11) lead to

$$
H^{\prime}=\frac{p^{2}}{2 m(t) e^{2 \epsilon(t)}}+V\left(e^{\epsilon(t)} x, t\right)-\frac{\dot{\epsilon}(t)}{2}\{x, p\}
$$

Therefore the transformed Hamiltonian is not of the standard form (12). It can however be put in this form by the canonical transformation defined by

$$
\mathcal{U}^{\prime}=\exp \left[\frac{-i}{2}\left(\dot{\epsilon} m e^{2 \epsilon}\right) x^{2}\right]
$$

This leads to

$$
\begin{aligned}
x \rightarrow x^{\prime \prime} & =x, \quad p \rightarrow p^{\prime \prime}=p+m e^{2 \epsilon} \dot{\epsilon} x, \\
H^{\prime} \rightarrow H^{\prime \prime} & =\frac{p^{2}}{2 m e^{2 \epsilon}}+V\left(e^{\epsilon} x, t\right)+\frac{1}{2}\left[\frac{d}{d t}\left(m e^{2 \epsilon} \dot{\epsilon}\right)-m e^{2 \epsilon} \dot{\epsilon}^{2}\right] x^{2} .
\end{aligned}
$$




\section{Time-Dependent Harmonic Oscillator}

The Hamiltonian of a time-dependent harmonic oscillator with mass $m=m(t)$ and frequency $\omega=\omega(t)$ is given by

$$
H=\frac{p^{2}}{2 m(t)}+\frac{1}{2} m \omega(t)^{2} x^{2} .
$$

For this system Eq.(16) takes the form

$$
H^{\prime \prime}=\frac{p^{2}}{2 m e^{2 \epsilon}}+\frac{1}{2}\left[\frac{d}{d t}\left(m e^{2 \epsilon} \dot{\epsilon}\right)+m e^{2 \epsilon}\left(\omega^{2}-\dot{\epsilon}^{2}\right)\right] x^{2},
$$

where time-dependence of $m, \omega$ and $\epsilon$ are suppressed for brevity.

Next let us choose $\epsilon(t)=\ln \left[m_{0} / m(t)\right] / 2$ for a positive constant $m_{0}$, so that $m e^{2 \epsilon}=m_{0}$. This choice yields

$$
H^{\prime \prime}=\frac{p^{2}}{2 m_{0}}+\frac{1}{2} m_{0} \Omega^{2} x^{2}
$$

which is the Hamiltonian for a harmonic oscillator with constant mass $m_{0}$ and frequency

$$
\Omega:=\sqrt{\ddot{\epsilon}-\dot{\epsilon}^{2}+\omega^{2}} .
$$

Requiring $\Omega$ to be independent of time, one can exactly solve the Schrödinger equation for $H^{\prime \prime}$ which is now time-independent. Using the canonical transformation defined by $\mathcal{U}^{\prime \prime}:=\left(\mathcal{U}^{\prime} \mathcal{U}\right)^{\dagger}$ and Eq. (3), one then finds the exact solution of the Schrödinger equation for the original harmonic oscillator. Thus the requirement $\Omega=\Omega_{0}=$ const. corresponds to a class of exactly solvable time-dependent harmonic oscillators. Note that although time-dependent harmonic oscillators have been extensively studied during the past five decades [1] - [14], for arbitrary

choices of mass $m(t)$ and frequency $\omega(t)$ a closed expression for the time-evolution operator in terms of $m(t)$ and $\omega(t)$ is not yet known.

Next let us re-express the condition $\Omega=\Omega_{0}$ in terms of $m$ and $\omega$. This leads to

$$
\ddot{\epsilon}-\dot{\epsilon}^{2}+\alpha^{2}=0, \quad \alpha:=\sqrt{\omega^{2}-\Omega_{0}^{2}},
$$

or alternatively

$$
\omega=\sqrt{\Omega_{0}^{2}+\frac{\ddot{m}}{2 m}-\left(\frac{\dot{m}}{2 m}\right)^{2}} .
$$

Hence, according to the above argument, the time-dependent oscillators whose mass $m$ and frequency $\omega$ satisfy (22) are canonically equivalent to the time-independent harmonic oscillator (19) with $\Omega=\Omega_{0}$. 
Next let us consider the case where the frequency $\omega$ is constant. Then Eq. (22) can be easily integrated to yield

$$
m(t)=m_{0}\left(\mu e^{\alpha t}+\nu e^{-\alpha t}\right)^{2}
$$

where $\mu$ and $\nu$ are constants. Clearly, the Caldirola-Kanai oscillator [1] whose mass depends exponentially on time, i.e., $m=m_{0} e^{\gamma t}$ belongs to this class of oscillators. In fact, Colegrave and Abdalla [12] have considered using the canonical transformation (10) to treat the oscillators with time-dependent mass and fixed frequency, and in particular the Caldirola-Kanai oscillator. However, they perform the canonical transformation within the classical context and then quantize the Hamiltonian. Fortunately the Hermiticity requirement determines

the quantum Hamiltonian uniquely. Hence the lack of knowledge about the precise unitary transformation corresponding to this canonical transformation does not play much of a role in their analysis.

\section{Quantum to Classical Reduction of the Dynamical Equation and the Ermankov Equation}

Consider the transformed harmonic oscillator Hamiltonian (18). If the square bracket on the right hand side of (18) vanishes, i.e.

$$
\frac{d}{d t}\left(m e^{2 \epsilon} \dot{\epsilon}\right)+m e^{2 \epsilon}\left(\omega^{2}-\dot{\epsilon}^{2}\right)=0
$$

then $H^{\prime \prime}$ describes a free particle with time-dependent mass. The corresponding Schrödinger equation is exactly solvable. This means that if one chooses $\epsilon$ such that the requirement (24) is satisfied, then one obtains the solution of the Schrödinger equation for the most general time-dependent harmonic oscillator.

Now if one introduces $\chi:=e^{\epsilon}$ and expresses Eq. (24) in terms of $\chi$, one obtains

$$
\frac{d}{d t}(m \dot{\chi})+m \omega^{2} \chi=0
$$

It is not difficult to recognize this equation as the classical equation of motion for the timedependent harmonic oscillator.

The reduction of the Schrödinger equation to the classical equation of motion is known 
since 1950's, [3, 7, 13]. It is nevertheless interesting to see that this reduction may be performed using a canonical transformation.

It should be emphasized that any positive real solution of Eq. (25) may be used to obtain the exact solution of the Schrödinger equation for the time-dependent harmonic oscillator (17). If a positive solution of (25) is found, then the time-evolution operator for the oscillator (17) is given by

$$
U(t)=\mathcal{U}(t)^{\dagger} \mathcal{U}(t)^{\dagger} V(t) \mathcal{U}^{\prime}(0) \mathcal{U}(0)
$$

where

$$
\begin{aligned}
\mathcal{U}(t) & :=e^{\frac{i}{2}(\ln \chi)\{x, p\}}, \\
\mathcal{U}^{\prime}(t) & :=e^{-\frac{i}{2}(m \chi \dot{\chi}) x^{2}}, \\
V(t) & :=e^{-i a(t) p^{2} / 2}, \\
a(t) & :=\int_{0}^{t} \frac{d t^{\prime}}{m\left(t^{\prime}\right) \chi\left(t^{\prime}\right)^{2}} .
\end{aligned}
$$

A slight generalization of condition (24) is to demand that the adiabatic approximation yields the exact solution of the Schrödinger equation for the transformed Hamiltonian (18). In view of the results reported in [14], the condition of the exactness of the adiabatic approximation for a time-dependent harmonic oscillator is that the product of its mass and frequecy be a constant $k$. For the oscillator (18), this means

$$
\left[\frac{d}{d t}\left(m e^{2 \epsilon} \dot{\epsilon}\right)+e^{2 \epsilon}\left(\omega^{2}-\dot{\epsilon}^{2}\right)\right] m e^{2 \epsilon}=k^{2} .
$$

In terms of the variable $\chi:=e^{\epsilon}$, Eq. (27) has the form

$$
\left[\frac{d}{d t}(m \dot{\chi})+m \omega^{2} \chi\right] m \chi^{3}=k^{2} .
$$

For $k=0$, it reduces to Eq. (25). For $k \neq 0$, a simple rescaling of $\chi$ by $\sqrt{|k|}$, namely $\chi \rightarrow \chi^{\prime}:=\chi / \sqrt{|k|}$, leads to

$$
\left[\frac{d}{d t}\left(m \dot{\chi}^{\prime}\right)+m \omega^{2} \chi^{\prime}\right] m \chi^{\prime 3}= \pm 1
$$

where the minus sign corresponds to the case where the transformed oscillator (18) has imaginary frequency. This means that the relevant values of $k^{2}$ in (28) are $-1,0$ and 1. 
Again any positive solution of any of the Eqs. (29) leads to the solution of the Schrödinger equation. The time-evolution operator is still given by Eq. (26), but now

$$
V(t):=e^{-i \alpha(t)\left(p^{2}+k^{2} x^{2}\right) / 2}
$$

Having obtained the expression for the time-evolution operator, one can easily compute the time-dependent position and momentum operators. The result is

$$
x(t)=a(t) x+b(t) p, \quad p(t)=c(t) x+d(t) p,
$$

where

$$
\begin{aligned}
a & :=\left(\frac{\chi}{\chi_{0}}\right)\left[\cos (k \alpha)-\frac{m_{0} \chi_{0} \dot{\chi}_{0} \sin (k \alpha)}{k}\right] \\
b & :=\frac{\chi_{0} \chi \sin (k \alpha)}{k} \\
c & :=\left(\frac{m \dot{\chi}}{\chi_{0}}-\frac{m_{0} \dot{\chi}_{0}}{\chi}\right) \cos (k \alpha)-\left(\frac{k}{\chi_{0} \chi}+\frac{m_{0} \dot{\chi}_{0} m \dot{\chi}}{k}\right) \sin (k \alpha), \\
d & :=\left(\frac{\chi_{0}}{\chi}\right) \cos (k \alpha)+\left(\frac{\chi_{0} m \dot{\chi}}{k}\right) \sin (k \alpha), \\
m_{0} & :=m(0), \quad \chi_{0}:=\chi(0), \quad \dot{\chi}_{0}:=\dot{\chi}(0) .
\end{aligned}
$$

The expressions corresponding to $k=0$ are obtained from (30) in the limit $k \rightarrow 0$. One can check that indeed $x(0)=x, p(0)=p$, and $[x(t), p(t)]=i$.

It is remarkable to note that using the (quadratic) invariant theory of Lewis and Riesenfeld [5], Lewis [4] had reduced the solution of the Schrödinger equation for a harmonic oscillator with time-dependent frequency to the solution of Eq. (28) with $k=1$. This equation was previously considered by Ermankov [15]. Here we obtained this equation by demanding that the adiabatic approximation yields the exact solution of the Schrödinger equation for the canonically transformed system.

One may generalize the results of this section by performing other time-dependent canonical transformations and demanding the result to be exactly solvable. For example by requiring the transformed Hamiltonian (18) to be one of the exactly solvable oscillators obtained in Ref. [14], one obtains various generalizations of Eq. (28). These are, however, integrodifferential equations whose solution seems to be at least as difficult as Eq. (28). 


\section{Time-dependent Diffeomorphisms which Change the Metric}

The time-dependent dilatations which correspond to the choice $f(x)=x$ in (1) form a very small class of quantum canonical transformations of the form (1). As it is seen from Eqs. (5) and (6) the transformations induced on the position and momentum operators depend in a complicated manner on $f(x)$. Some other choices of $f(x)$ for which these transformations can be calculated in a closed form are

$$
\begin{aligned}
& f(x)=x^{2}: \quad\left\{\begin{array}{llc}
x & \rightarrow & x^{\prime}=\frac{x}{1-\epsilon(t) x} \\
p & \rightarrow & p^{\prime}=[1-\epsilon(t) x] p[1-\epsilon(t) x]
\end{array}\right\} \quad \text { for } \quad|\epsilon(t) x|<1 \\
& f(x)=e^{-\lambda x}:\left\{\begin{array}{llc}
x & \rightarrow & x^{\prime}=\frac{1}{\lambda} \ln \left[e^{\lambda x}+\epsilon(t) \lambda\right] \\
p & \rightarrow & p^{\prime}=\sqrt{1+\frac{\epsilon(t) e^{\lambda x}}{\lambda}} p \sqrt{1+\frac{\epsilon(t) e^{\lambda x}}{\lambda}}
\end{array}\right\} \text { for }|\epsilon(t) \lambda|<1,
\end{aligned}
$$

where $\lambda$ is a positive real number. As seen from these formulae, the effect of these canonical transformations on the kinetic part $p^{2} / 2 m$ of the Hamiltonian is to make the mass $m$ also depend on the position. This is precisely what happens when one considers a free particle moving on a line with a metric $g$. In this case the quantum Hamiltonian is given by 16]

$$
\mathcal{H}=\frac{1}{2 m}\left[g^{-1 / 4} p g^{-1 / 2} p g^{-1 / 4}\right] \text {. }
$$

It is uniquely determined by the classical Hamiltonian $\mathcal{H}_{c}=p^{2} /(2 m g)$ and the self-adjointness requirement with respect to the measure $\sqrt{g} d x$.

In view of Eqs. (32) and (6), one can easily infer the fact that under the canonical transformations (1) a free particle in $\mathbb{R}$ with the metric $g(x)=1$ is mapped to a free particle in $\mathbb{R}$ with a metric $g=\left[\mathcal{F}_{2}\right]^{-2}$. This is precisely the metric one would obtain by performing the diffeomorphism $x \rightarrow x^{\prime}=\mathcal{F}_{1}$. The converse of this statement is also true in the sense that for an arbitrary metric $g=g(x ; t)$, there is a canonical transformation of the form (1) which maps the problem to the ordinary one-dimensional free particle problem provided that one can solve the pseudo-differential equation

$$
f(x) e^{\epsilon(t) f(x) \frac{d}{d x}} f^{-1}(x)=: \mathcal{F}_{2}(x)=\left[g\left(\mathcal{F}_{1}(x) ; t\right)\right]^{-1 / 2}:=\left[g\left(e^{\epsilon(t) f(x) \frac{d}{d x}} x ; t\right)\right]^{-1 / 2}
$$

for $f(x)$. For the examples listed in (31), one has

$$
f=x^{2} \quad \Longleftrightarrow g=[1-\epsilon(t) x]^{-4}
$$




$$
f=e^{-\lambda x} \Longleftrightarrow g=\left[1+\frac{\epsilon(t) e^{\lambda x}}{\lambda}\right]^{-2}
$$

These considerations show that the one-dimensional quantum mechanics of a free particle with position (and time) dependent mass is canonically equivalent to that of a free particle with constant mass.

\section{Conclusion}

In this article I have explored the quantum canonical transformations corresponding to time-dependent diffeomorphisms of the configuration space $\mathbb{R}$. A special class of these transformations which are associated with the time-dependent dilatations is used to obtain a new class of exactly solvable time-dependent harmonic oscillators. A well-known oscillator which belongs to this class is the Caldirola-Kanai oscillator.

Another application of time-dependent dilatations is in the reduction of the Schrödinger equation for the general time-dependent harmonic oscillator to the corresponding classical equation of motion. Although the relation between the quantum and classical dynamical equations has been well-known, its direct realization via time-dependent dilatations is a new result. More specifically, I have shown that

- if one uses a positive solution $\chi=\chi(t)$ of the classical equation of motion (25) to perform the time-dependent quantum dilatation $x \rightarrow \chi(t) x$, then one obtains the solution of the Schrödinger equation;

- if one performs a quantum dilatation $x \rightarrow \chi(t) x$, canonically transforms the resulting generalized harmonic oscillator Hamiltonian to a Hamiltonian of the standard form, and requires the adiabatic approximation to yield the exact solution of the Schrödinger equation for the latter Hamiltonian, then one obtains a second order differential equation in the dilatation parameter $\chi$ which involves a parameter $k^{2}=-1,0,1$. For $k=0$, this is the classical equation of motion. For $k=1$, this is known as the Ermankov equation which is also obtained by applying the Lewis-Riesenfeld invariant theory to the time-dependent harmonic oscillator. 
I have also briefly commented on the quantum canonical transformations which correspond to metric-changing diffeomorphisms.

The direct generalization of the analysis presented in this article to higher dimensions is not difficult. In fact, the $n$-dimensional analogue of (1), i.e.,

$$
\mathcal{U}:=\exp \left[\frac{i \epsilon(t)}{2} \sum_{i=1}^{n}\left\{f^{i}(x), p_{i}\right\}\right]=\exp \left[i \epsilon(t) \sum_{i=1}^{n} \sqrt{f^{i}(x)} p_{i} \sqrt{f^{i}(x)}\right]
$$

corresponds to diffeomorphisms of $\mathbb{R}^{n}$. Here $x=\left(x^{1}, \cdots, x^{2}\right)$ and $f^{i}(x)$ are smooth realvalued functions of $x$. For $n>1$ there are metrics on $\mathbb{R}^{n}$ which are not related to the Euclidean metric by a diffeomorphism. Thus, in general canonical transformations of the form (33) do not relate the dynamics of a free particle on an arbitrary curved $\mathbb{R}^{n}$ to that of $\mathbb{R}^{n}$ with Euclidean geometry.

\section{Acknowledgments}

Parts of this project were completed while I was a member of the Theoretical Physics Institute of the University of Alberta. I wish to acknowledge the financial support of the Killam Foundation of Canada and thank Drs. M. Razavy and B. Darian for interesting discussions.

Note: After the completion of this article Refs. [17, 18] were brought to my attention. In Ref. [17] the author uses the quantum dilatations (10) to study the solution of the Schrödinger equation with a moving boundary condition. Ref. 18 gives a systematic analysis of the most general exponential quadratic operator in $x$ and $p$, whose special case is the quantum dilatations studied in this article.

\section{References}

[1] P. Caldirola, Nuovo Cimento 18, 393 (1941);

E. Kanai, Prog. Theo. Phys. 3, 440 (1948).

[2] W. E. Brittin, Phys. Rev. 77, 396 (1950).

[3] K. Husimi, Prog. Theo. Phys. 9, 381 (1953). 
[4] H. R. Lewis, Jr. Phys. Rev. Lett. 18, 510 (1967).

[5] H. R. Lewis, Jr. and W. B. Riesenfeld, J. Math. Phys. 10, 1458 (1969).

[6] I. A. Malkin and V. I. Man'ko, Phys. Lett. A 32, 243 (1970).

[7] I. A. Malkin, V. I. Man'ko, and D. A. Trifonov, J. Math. Phys. 14, 576 (1973)

[8] D. M. Greenberger, J. Math. Phys. 15, 395 (1974);

R. W. Hasse, J. Math. Phys. 16, 2005 (1975);

V. V. Dodonov and V. I. Man'ko, Phys. Rev.A 20, 550 (1979);

L. F. Landovitz, A. M. Levine and W. M. Schreiber, Phys. Rev. A 20, 1162 (1979);

M. S. Abdalla and R. K. Colegrave, Phys. Rev. A 32, 1958 (1985);

A. B. Nassar and R. T. Berg, Phys. Rev. A 34, 2462 (1986);

S. V. Prants, J. Phys. A: Math. Gen. 19, 3457 (1986);

C. M. Cheng and P. C. W. Fung, J. Phys. A: Math. Gen. 21, 4115 (1988);

F. Wolfs and H. J. Korsch, Phys. Rev. A 37, 1934 (1988);

A. de Souza Dutra, C. F. de Souza, and L. C. de Albuquerque, Phys. Lett. A 156, 371 (1991);

S. P. Kim, J. Phys. A: Math. Gen. 27, 3927 (1994);

S. J. Wang, W. Zuo, A. Weiguny, and F. L. Li, Phys. Lett. A 196, 7 (1994);

Y.-Z. Lai, J.-Q. Liang, H. J. W. Müller-Kirsten, and J.-G. Zhou, Phys. A: Math. Gen. 29, 1773 (1996);

Sh. Zhang and F. Li, J., Phys. A: Math. Gen. 29, 6143 (1996);

H.-C. Kim, M.-H. Lee, J.-Y. Ji, and K. Kim, Phys. Rev. A 53, 3767 (1996);

V. Penna, Ann. Phys. 245, 389 (1996);

Ch.-I. Um, I.-H. Kim, K.-H. Yeon, T. F. George, and L. N. Pandey, Phys. A: Math. Gen. 30, 2545 (1997).

[9] W. Paul, Rev. Mod. Phys. 62, 531 (1990).

[10] F. Diedrich, J. C. Bergquist, W. M. Itano, and D. J. Wineland, Phys. Rev. Lett. 62 403 (1989);

D. J. Heinzen and D. J. Wineland, Phys. Rev. A 42, 2977 (1990). 
[11] G. S. Agarwal and S. Arun Kumar, Phys. Rev. Lett. 67, 3665 (1991).

[12] R. K. Colegrave and M. Sebawe Abdalla, J. Phys. A: Math. Gen. 14, 2269 (1981); M. Sebawe Abdalla and R. K. Colegrave, Lett. Nuovo Cimento 39, 1111 (1984); M. Sebawe Abdalla, Phys. Rev. A 33, 2870 (1986).

[13] J.-Y. Ji, J. K. Kim, and S. P. Kim, Phys. Rev.A 51, 4268 (1995).

[14] A. Mostafazadeh, Phys. Rev. A 55, 4084 (1997).

[15] V. P. Ermankov, Univ. Izv. Kiev. 20, 1 (1880).

[16] B. S. DeWitt, Supermanifolds, (Cambridge University press, Cambridge, 1992).

[17] M. Razavy, Phys. Rev. A 8, 153 (1983).

[18] X.-B. Wang, C. H. Oh, and L. C. Kwek, quant-ph/982048. 